\title{
ESCRITURA ACADÉMICA PARA EL DESARROLLO DE LA REFLEXIÓN PEDAGÓGICA EN LA FORMACIÓN DOCENTE: LA CRÓNICA DEL DOCENTE-DIRECTIVO
}

\author{
ACADEMIC WRITING FOR THE DEVELOPMENT OF PEDAGOGICAL REFLECTION IN TEACHER \\ education: A Chronicle Teacher-Headmaster
}

ÉCRITURE ACADÉMIQUE POUR LE DÉVELOPPEMENT DE LA RÉFLEXION PÉDAGOGIQUE DANS LA FORMATION DES ENSEIGNANTS: LA CHRONIQUE ENSEIGNANT-DIRECTEUR

\author{
Marcela Jarpa-Azagra \\ Dra. en Lingüística, Pontificia \\ Universidad Católica de Valparaíso, \\ Chile. Profesora asociada, jefa de \\ investigación y estudios avanzados, \\ Escuela de Pedagogía, Pontificia \\ Universidad Católica de Valparaíso, \\ Chile. \\ Avenida El Bosque 1290, 3. er piso, \\ edificio B, Sausalito, Viña del Mar, \\ Chile. \\ marcela.jarpa@pucv.cl \\ https://orcid.org/0000-0003- \\ 4171-3085
}

\begin{abstract}
RESUMEN
El artículo presenta los principales resultados de una investigación-acción que tuvo como eje el diseño e implementación de un módulo de enseñanza de la escritura académica para potenciar el desarrollo de la reflexión profesional docente en un programa de posgrado de educación en una universidad chilena. El objetivo general fue definir y caracterizar un nuevo género académico de reflexión pedagógica que permitiera el desarrollo de una escritura epistémica (Bereiter y Scardamalia, 1987) para potenciar el saber pedagógico de los docentes. El diseño metodológico de esta investigación contempló un enfoque teórico-práctico y de carácter longitudinal, que permitió la participación de 160 docentes y la conformación de un corpus de análisis de 160 textos recogidos en un periodo de 5 años. Entre los principales resultados se destaca, en primer lugar, la creación de un género de reflexión docente al que hemos denominado "crónica del docente-directivo", cuyo escrito permite un análisis y la evaluación profunda del desempeño profesional, el que se sistematiza a partir de la organización retórica de las movidas del género. En segundo lugar, el diseño e implementación de una secuencia didáctica basada en la pedagogía del género, que posibilita el andamiaje y el desarrollo de la escritura reflexiva como un recurso profesional que potencia el saber docente. Una conclusión relevante es que el proceso de modelaje y andamiaje de este género permite mejorar no solo las competencias comunicativas de los docentes, sino también la profundidad de la reflexión pedagógica, logrando la construcción y la consolidación del saber docente de cada uno de los profesores participantes.
\end{abstract}

Palabras clave: escritura académica; géneros de reflexión pedagógica; pedagogía del género; saber pedagógico; movidas retóricas.

\section{Abstract}

The paper presents the main results of an action research that had as its axis the design and implementation of a teaching module of academic writing to promote the development of teacher professional reflection in a postgraduate education program in a Chilean university. The aim was to define and characterize a new academic genre of pedagogical reflection that allowed the development of an epistemic writing to enhance the pedagogical knowledge of teachers. The methodological design of this research contemplated a theoretical-practical and longitudinal approach that allowed

Received: 2017-11-13 / Accepted: 2018-08-02 / Published: 2019-01-24

DOI: $10.17533 /$ udea.ikala.v24n01a04 
the participation of 160 teachers and the formation of a corpus of analysis of 160 texts collected in a period of 5 years. Among the main results, we highlight, firstly, the creation of a kind of teacher reflection that we have called Chronicle TeachingDirective that allows a deep analysis of the practice from the rhetorical organization of the moves. Secondly, the design and implementation of a didactic sequence based on the pedagogy of the genre that allows the scaffolding and the development of reflexive writing as a professional resource that potentiates teacher's knowledge is highlighted. A relevant conclusion is that the modeling and scaffolding process of this genre allows us to improve not only the communicative competences of the teachers but also the depth of the pedagogical reflection, achieving the construction and consolidation of the teaching knowledge of each of the participating teachers.

Keywords: academic writing; pedagogical reflexión genre; genre of pedagogy; pedagogical knowledge; rhetorical move.

\section{RÉSUMÉ}

L'article présente les principaux résultats d'une recherche-action a été axée sur la conception et la mise en œuvre d'un module d'enseignement écriture académique pour améliorer le développement professionnel de la réflexion de l'enseignement dans un programme d'études supérieures à une formation universitaire chilienne. L'objectif général était de définir et de caractériser un nouveau genre académique de réflexion pédagogique qui permettrait le développement d'une écriture épistémique pour améliorer les connaissances pédagogiques des enseignants. La conception méthodologique de cette recherche a examiné un caractère théorique et pratique et l'approche longitudinale a permis la participation de 160 enseignants et la création d'un corpus d'analyse de 160 textes recueillis sur une période de 5 ans. Parmi les résultats principaux ont émergé, d'abord, en créant une sorte de réflexion des enseignants que nous avons appelé l'enseignant-directive chronique permettant une analyse approfondie de la pratique de l'organisation rhétorique des mouvements. En second lieu, la conception et la mise en œuvre d'une séquence d'enseignement basée sur la pédagogie du genre qui permet à l'échafaudage et le développement de l'écriture réfléchissant comme une ressource professionnelle qui améliore l'enseignant est soulagé savent. Une conclusion pertinente est que le processus de modélisation et d'échafaudage de ce genre nous permet d'améliorer non seulement les compétences communicatives des enseignants, mais également la profondeur de la réflexion pédagogique, permettant ainsi de construire et de consolider les connaissances pédagogiques de chacun des enseignants participants.

Mots clés : écriture académique ; genre de réflexion pédagogique ; genre de pédagogie; connaissances pédagogiques; mouvement rhétorique. 


\section{Introducción}

La literatura especializada e investigaciones en el ámbito del desarrollo profesional docente (DarlingHammond, 2006, 2012) confirman que existe una relación directa entre la calidad de la formación y el desempeño de los profesores en contextos escolares. Por esta razón, resulta muy relevante que los programas de pregrado, posgrado y formación continua posean dispositivos y recursos eficaces para fortalecer el saber pedagógico de los maestros, articulando la teoría y la práctica.

Esta articulación discursiva entre conocimiento especializado y práctica es un aspecto complejo que dificulta el acceso a las comunidades de posgrado, fundamentalmente porque los profesores tienen prácticas discusivas diferentes en los contextos escolares donde se desempeñan. La escritura académica es una habilidad que implica nuevas exigencias para el maestro, puesto que estos géneros discursivos escritos se caracterizan por incorporar no solo un complejo componente analítico, crítico y reflexivo, sino también nuevas formas de comunicar lo aprendido. Si bien es cierto que estas condiciones permiten construir y consolidar el conocimiento disciplinar, asimismo se constituyen en obstáculos para la trayectoria formativa de los docentes, en tanto que cuentan con poca experiencia en la escritura de este tipo de géneros.

Bajo este escenario, el equipo de académicos del Magíster en Liderazgo y Gestión de Organizaciones Escolares, de la Pontificia Universidad Católica de Valparaíso (Chile), decidió incorporar un módulo de escritura académica que permitiera, a los estudiantes, enfrentar las dificultades que tenían para escribir un texto académico y que integrara el análisis de fuentes bibliográficas con la evaluación y la reflexión crítica de sus propias experiencias profesionales. Por lo tanto, el eje formativo de este módulo fue la enseñanza de un nuevo género de reflexión pedagógica denominado "crónica del docente-directivo", que pudiera vincular el conocimiento especializado que entregaba el Magíster con la experiencia práctica de los docentes. La principal tarea del módulo consistía en que los estudiantes aprendieran a escribir este género, mediante el seguimiento de ciertas orientaciones conceptuales y prácticas, y así andamiar, paulatinamente, la adquisición de habilidades de escritura académica.

La crónica del docente-directivo forma parte de la familia de géneros de reflexión profesional docente, y tiene como propósito comunicativo evaluar críticamente la propia experiencia docente, a partir de la identificación de una disonancia cognitiva, esto es, tensión o desarmonía en su sistema de creencias, ideas o emociones (Festinger, 1957), aspecto central que posteriormente le permitirá movilizar su conocimiento especializado en pos de una apropiación conceptual que consolide su saber pedagógico.

El enfoque metodológico de esta experiencia se abordó desde la investigación-acción, razón por la cual académicos y estudiantes del programa adquirieron un rol protagónico, pues en conjunto desarrollaron la construcción y la consolidación de un nuevo género de reflexión pedagógica, con el que pudieron identificar una disonancia cognitiva en su experiencia profesional, anclar un tópico disciplinar y andamiar un modelo de reflexión crítica. De esta manera, se diseñó e implementó una intervención didáctica desde la pedagogía del género (Chaisiri, 2010), condición que permite asumir la escritura académica como una herramienta fundamental para dar cuenta de esta reflexión.

Un elemento relevante de este proceso es el acompañamiento a los escritores, mediante borradores y retroalimentaciones personalizadas, que posibilitan el desarrollo de una escritura de carácter más académico, formalizando un tipo de discurso propio que da cuenta de las demandas y convenciones de la comunidad pedagógica donde se inserta el programa de posgrado.

En los apartados posteriores revisamos los principales supuestos teóricos que sustentaron esta investigación, como también los procedimientos metodológicos utilizados tanto para el diseño de 
la intervención didáctica, como para el análisis del corpus. Finalmente, se presentan los resultados, relevando dos aspectos: el modelo de secuencia didáctica implementado, y el producto asociado a la definición y la caracterización de un nuevo género para la reflexión pedagógica.

\section{Marco teórico}

El marco teórico de esta investigación se basa en tres perspectivas conceptuales: teoría del género, pedagogía del género y reflexión pedagógica.

La teoría de género permite la definición y la caracterización del nuevo género (Jarpa, 2012; Parodi, Ibáñez, Venegas y González, 2010; Swales, 2000), mediante la sistematización de sus rasgos léxicogramaticales y retórico-estructurales, con el fin de obtener una respuesta relativamente convencional a una situación comunicativa específica. En cuanto a la pedagogía del género (Chaisiri, 2010; Hyland, 2003), esta andamia todo el modelo implementación didáctica del módulo de escritura. Finalmente, la reflexión pedagógica (Jay y Johnson, 2002; Schön, 1998; Villalobos y De Cabrera, 2009) es la base del modelo analítico-evaluativo del género, que aporta los tópicos y el nivel de reflexión para la elaboración y la construcción del saber pedagógico de los docentes.

\section{Aspectos conceptuales y metodológicos para la conformación de un nuevo género de reflexión pedagógica}

Una forma de estudiar el desarrollo y el ejercicio de la reflexión pedagógica es mediante la identificación de los géneros que se escriben en la formación docente. La escritura, además de ser una herramienta epistémica, es decir, permite la construcción de conocimiento académico (Carlino, 2006; Cassany, 2006), y de aprendizaje, también es un medio que potencia el desarrollo del pensamiento reflexivo (Bereiter y Scardamalia, 1987; Yinger y Clark, 1981), y se constituye en un elemento de vital importancia para evaluar el desempeño del profesor,

no solo al interior de aula, sino también en otros contextos formativos (Korthagen, 2004; Quintero, Torres y Cardona, 2007; Starks, Nicholas y Macdonald, 2012). Por esta razón, surge la necesidad de identificar, definir y caracterizar aquellos géneros que circulan en la formación inicial y continua de profesores, y para tal efecto es útil considerar los aportes provenientes de la teoría del género (Bazerman, 2004; Hyland, 2007; Swales, 2000).

De aquí que, al momento de definir un género, sea posible reconocer algunas perspectivas que se centran en el contexto social y comunicativo, y otras que ponen de relieve las características internas comunes que existen para un determinado grupo de textos. La definición que tiene una visión integradora y que abarca tanto el enfoque intratextual como el extratextual es la de Swales (2000), pues no solo toma en cuenta las características internas convencionales, sino también la idea de que existen prototipos textuales compartidos por los miembros expertos de una comunidad discursiva y que son utilizados en determinados contextos comunicativos. Siguiendo esta perspectiva, Bhatia (1993) amplía y profundiza el concepto, al incorporar la intención comunicativa como un factor principal para caracterizar los géneros y su estructura interna, lo que permite distinguir y crear géneros y subgéneros al interior de una comunidad discursiva.

Para Bhatia (1993), la finalidad comunicativa se refleja inevitablemente en la estructuración cognitiva interpretativa del género, lo que manifiesta una estrecha relación entre la intención comunicativa y las formas de organización prototípicas que tiene una determinada comunidad discursiva. Tales regularidades de organización estructural deben ser consideradas como cognitivas, porque reflejan las estrategias usadas por los miembros de una comunidad discursiva, tanto para la construcción y el entendimiento de un género, como para alcanzar una finalidad comunicativa específica.

Para llevar a cabo una interpretación estructural de un género textual es necesario poner de 
relieve los aspectos cognitivos de la organización del lenguaje, pues "in order to realize a particular communicative intention at the level of a move, an individual writer may use different rhetorical strategies" (Bhatia, 1993, p. 30). El autor distingue dos elementos retóricos, y los define como "discriminative elements of generic structure", y a las estrategias como "non-discriminative options within the allowable contributions available to an author for creative or innovative genre construction" (Bhatia, 1993, p. 30).

A partir de estas concepciones se ha desarrollado un método de análisis para la estructura retórica de los géneros, que busca determinar la organización macroestructural de un texto por medio de la segmentación de este en movidas y pasos. La movida corresponde a segmentos textuales que desarrollan un determinado tópico asociado a un propósito comunicativo. Por su parte, el paso correspondería a la unidad inmediatamente inferior a la movida, pues se constituye en un subtópico asociado al mismo propósito comunicativo. En su conjunto, movidas y pasos construyen la lógica textual y el significado global de un género, permitiendo una transposición didáctica desde los aspectos cognitivos del género a la concretización discursiva de este.

Por lo tanto, la movida retórica permite la identificación de ciertos patrones de organización discursiva de un género, dando cuenta no solo de la configuración textual, sino además de la forma como una determinada comunidad organiza, construye y divulga su conocimiento.

En este sentido, cada comunidad valida y reconoce los géneros especializados que circulan en su interior; por lo mismo, es posible identificar dos métodos de implantarlos, socializarlos e investigarlos: el primero tiene un carácter deductivo, pues releva la experiencia de los miembros expertos o con mayor jerarquía al interior de la comunidad, quienes definen y establecen aquellos géneros que son útiles para comunicar el conocimiento disciplinar o profesional. Este método implica la selección a priori de determinados géneros, los que entran en circulación según las necesidades y los propósitos comunicativos de la comunidad. El segundo método tiene una orientación inductiva o ecológica, ya que recopila los géneros que circulan de manera natural o espontánea en la comunidad y se construye un corpus, a partir del cual se hacen análisis, clasificaciones y se definen patrones comunes, para dar cuenta de los géneros que circulan y de la organización retórica de estos (Cotos, Huffman y Link, 2017; Kanoksilapatham, 2007).

Ya que el foco de esta investigación es definir y describir un género de reflexión pedagógica, ha sido necesario establecer ciertos criterios y variables que permitan constituir su propósito comunicativo y su organización retórica. Para ello se tuvieron en cuenta criterios provenientes de investigaciones preliminares (Jarpa, 2012; Parodi et al., 2010), como son: la relación entre los participantes, el contexto de circulación, el propósito comunicativo (Bhatia, 1993; Swales, 1990), el objetivo de aprendizaje (Anderson y Krathwohl, 2001), la modalidad (Kress y Van Leeuwen, 2001) y el modo de organización del discurso (Adam, 1992).

El conjunto de estas perspectivas teóricas ha permitido configurar este nuevo género y propiciar no solo un ámbito de investigación que promueve la escritura como un recurso de reflexión pedagógica, sino también constituir una comunidad de aprendizaje de posgrado (Bermúdez, 2009), con sus géneros prototípicos, y que contribuyen a fortalecer la formación académica de los docentes.

\section{El desarrollo de la escritura para la reflexión pedagógica desde una pedagogía basada en el género}

En la última década han aumentado las investigaciones y los trabajos que sitúan el concepto de género discursivo como un dispositivo fundamental para la enseñanza y el aprendizaje de la escritura (Bazerman, 2004; Hyland, 2003; Thaiss y Myers, 2006), pues aporta una perspectiva más precisa y funcional respecto de cómo la lengua es 
estructurada según los propósitos comunicativos y sociales de cada comunidad discursiva.

La pedagogía basada en el género se ha convertido en una corriente de estudio teórico y práctico que ofrece las herramientas necesarias para enseñar a escribir, tanto en contextos educativos, como profesionales (Chaisiri, 2010; Hyland, 2007; Venegas, Núñez, Zamora y Santana, 2015). Bajo este enfoque, se destaca la enseñanza de la escritura desde una perspectiva de proceso, la cual es asistida por especialistas o tutores, y acompañada con estrategias didácticas que andamian gradualmente la organización retórica y los rasgos lingüísticos del género, con el fin de que los escritores produzcan textos comprensivos y ajustados a las convenciones de su comunidad.

Este proceso de andamiaje de los géneros es necesario, ya que cada disciplina ha organizado distintos esquemas de pensamiento que se materializan y divulgan mediante variadas prácticas discursivas de escritura; por consiguiente, los sujetos que forman parte de estas comunidades deben conocer cada una de estas estructuras, aprehenderlas y utilizarlas adecuadamente. $\mathrm{Al}$ respecto, el saber profesional y la formación docente se constituyen como una disciplina y un campo de conocimiento especializado que atraviesa y potencia la formación de profesores. Esta comunidad posee modos específicos de comunicación, prácticas discursivas, propósitos comunicativos y objetivos de aprendizaje que configuran una cultura específica.

Dado que la escritura permite la transformación de la actividad docente en actividad intelectual (Quintero, Torres y Cardona, 2007), un aspecto importante de un profesor altamente capacitado es que sea también un escritor cualificado. Es por esto por lo que Zuluaga y Echeverri (2003) manifiestan que las instituciones educativas deben ser campos de experimentación pedagógica, donde los profesores estén llamados a integrar todas aquellas experiencias que están dispersas, siendo el ejercicio de la escritura y la reescritura una forma de vincular la teoría y la práctica, la investigación y la enseñanza.

No obstante, alcanzar el desarrollo de esta habilidad es complejo. Así lo corroboran Hatton y Smith (1995), quienes recogen un corpus de textos escritos al interior de un programa de formación de profesores y reconocen que un aspecto problemático es la forma como se promueve y alcanza el desarrollo de la reflexión en las prácticas pedagógicas. Para dar cuenta de esta realidad, una estrategia utilizada fue la escritura de diversos textos, entre los cuales se identificaron cuatro tipos de modalidades de escritura: la escritura descriptiva, la reflexión descriptiva, la reflexión dialógica y la reflexión crítica. Cada uno de estos tipos de reflexión escrita presenta diversos contenidos, modalidades discursivas, rasgos estilísticos, léxicos y gramaticales, que exponen la naturaleza de la reflexión y el nivel que se ha alcanzado.

Por lo tanto, para fortalecer la formación docente, tanto en pregrado como en posgrado y en formación continua, es necesario elevar el estatus y el rol de una escritura que promueva la reflexión pedagógica. Creemos que para alcanzar resultados significativos y trascendentes es necesario que los programas que forman profesores incorporen ejes curriculares basados en la escritura y asociados a determinados enfoques didácticos, como, por ejemplo, la pedagogía basada en el género (Chaisiri, 2010; Hyland, 2007), pues entrega un conjunto de prácticas de enseñanza donde el aprendiz está dentro de un marco contextual que le permite resignificar los diversos tipos de textos que circulan en una determinada situación comunicativa.

Para alcanzar esta meta es necesario que los estudiantes tomen conciencia de los patrones léxico-gramaticales y retóricos que confluyen en estos textos, mediante una interacción colaborativa y progresiva entre el profesor y el estudiante, que permita el andamiaje contextual y cognitivo del género. 


\section{Marco metodológico}

El enfoque de esta investigación cualitativa se basa en los fundamentos de la investigaciónacción (Elliott, 1993; Kemmis y McTaggart, 1988; Lewin, 1973) y tiene como finalidad actuar sobre un problema determinado. Por lo mismo, la teoría emerge contextualizada, lo que permite sostener e intervenir las prácticas sociales de la comunidad con la que se trabaja (Williamson, 2002).

Algunos de los aspectos contextualizados que delimitaron la aplicación de esta metodología fueron:

1. El problema fue diagnosticado por la propia comunidad del programa de posgrado en el primer semestre del año 2013.

2. La búsqueda de una solución permitió mejorar las competencias profesionales de los estudiantes del programa de posgrado.

3. La propuesta de mejora y la ejecución de la misma fue realizada por integrantes del equipo de académicos del programa y por los propios estudiantes.

4. El impacto de las decisiones y los ajustes realizados contribuyeron al mejoramiento del programa y al fortalecimiento del equipo.

Estas condiciones fueron sistematizadas en un modelo genérico de espiral de ciclos sucesivos, entre los que se encuentran: "Diagnóstico", "Planificación", "Acción”, "Observación” y "Reflexión-Evaluación" (Elliott, 1993; Kemmis y McTaggart, 1988; Lewin, 1973). La figura 1 expone los ciclos contemplados en el presente estudio.

Estos ciclos son:

- Diagnóstico: se necesita generar un producto y una estrategia didáctica que mejore la escritura de los estudiantes del programa de posgrado, y que, además, desarrolle la reflexión pedagógica.

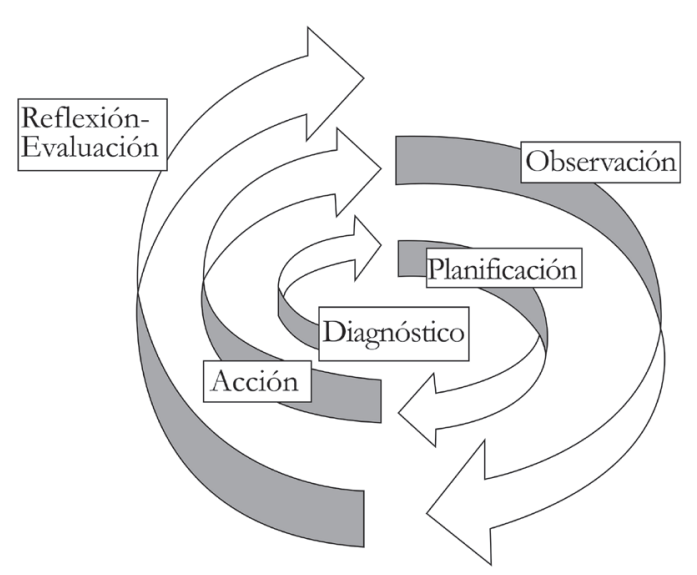

Figura 1. Espiral de ciclos de la investigación

- Planificación: se diseña un género nuevo denominado "crónica docente-directiva", para trabajar en el módulo de escritura académica, que permita articular la experiencia profesional y los nuevos conocimientos adquiridos en el programa de posgrado.

- Acción: se ejecuta un módulo de escritura académica bajo la perspectiva de la pedagogía del género, que posibilite un acompañamiento constante en el proceso de escritura, y con énfasis en la retroalimentación y la mediación.

- Observación: se observa, durante la implementación, la dificultad de los estudiantes del programa para escribir el género, sobre todo en los apartados que dan cuenta de la reflexión profesional y de la fundamentación teórica.

- Reflexión-Evaluación: se propone incorporar, en el programa de posgrado, de forma progresiva y permanente, el módulo de escritura académica, para que sea posible andamiar la reflexión profesional y su articulación con los conocimientos disciplinares del programa de posgrado.

Este análisis del contexto permitió establecer el objetivo general de la investigación-acción, que fue diseñar una intervención didáctica para potenciar las habilidades de escritura académica y la reflexión 
pedagógica de los docentes del programa de Posgrado en Educación.

En cuanto a los objetivos especificos, apuntaron a:

1. Establecer un género de reflexión pedagógica que responda a los requerimientos de escritura académica del programa de posgrado.

2. Describir la organización retórica del género de reflexión pedagógica, mediante la identificación de los segmentos textuales que lo configuran.

3. Implementar una secuencia didáctica que apoye la escritura del género de reflexión pedagógica establecido.
La duración total de esta investigación fue de cinco años, pues abarcó desde la primera cohorte, que ingresó el año 2012, hasta la cohorte de 2017, esto con la finalidad de observar el comportamiento del género y su potencialidad para desarrollar la reflexión pedagógica escrita de los docentes. Por esta razón, se cuenta con un corpus de 160 textos, los que corresponden al número total de docentes participantes en el programa durante el transcurso de estos años.

La figura 2 esquematiza la secuencia de actividades didácticas aplicadas para que los docentes lograran producir mejores textos académicos. El diseño de la intervención didáctica contempló una primera etapa de carácter deductiva, que implicó el modelamiento del género, mediante la ejecución

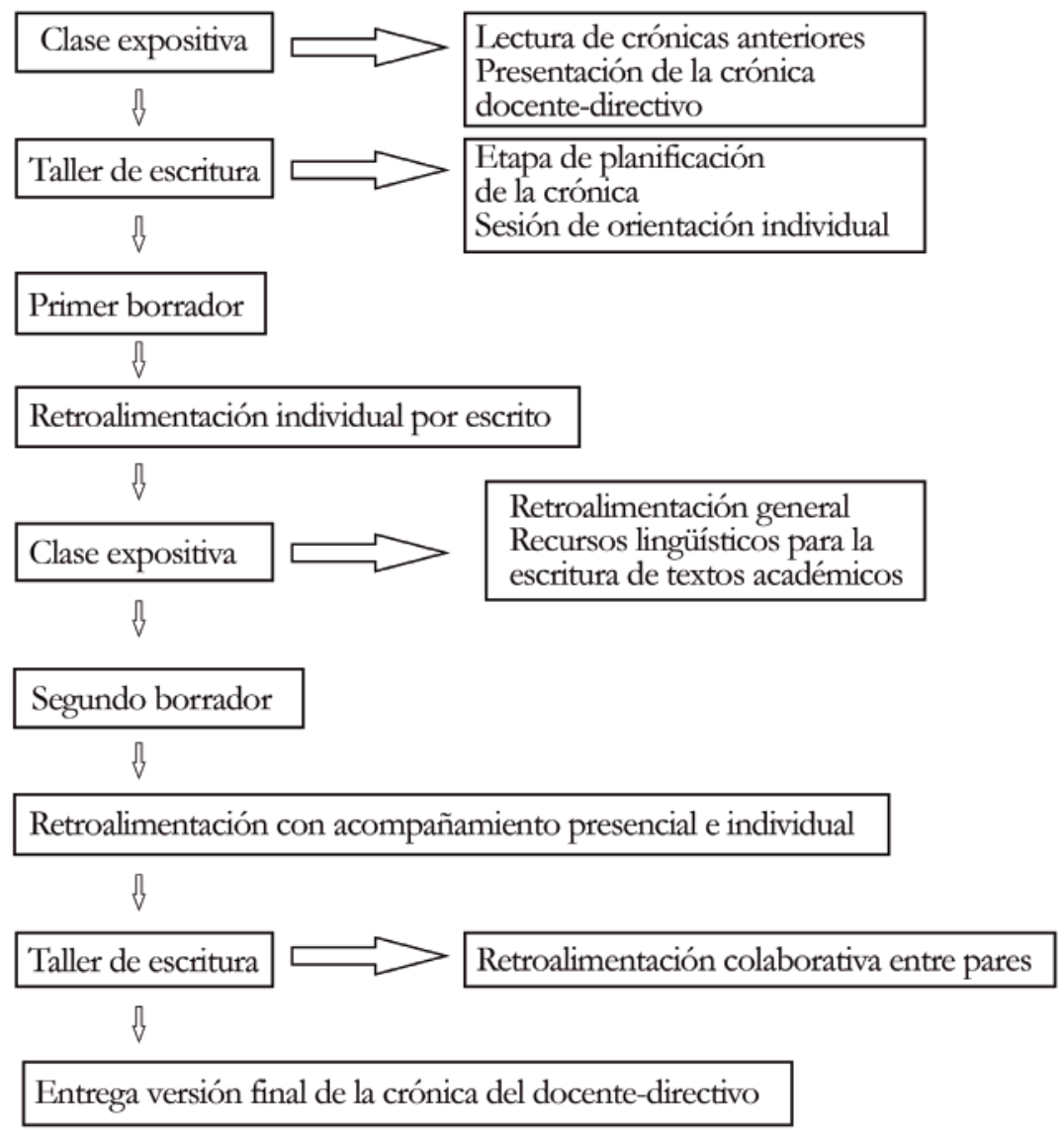

Figura 2 Secuencia didáctica del módulo de escritura de la crónica docente-directiva 
de un módulo de escritura académica con sesiones teórico-prácticas. La segunda etapa consistió en la recolección de los textos escritos por los docentes (con posterioridad a la intervención didáctica y asumiendo el último borrador como editado y entregado para calificar), para la conformación del corpus que permitiera evaluar el desarrollo de la reflexión pedagógica alcanzado y el comportamiento del género según este propósito. Para ello fue necesario analizar el nivel de dificultad, coincidencia y pertinencia de cada movida de la organización retórica del género propuesto.

En virtud de la experiencia recabada durante la ejecución de los talleres de escritura académica, la organización retórica del género fue ajustada en dos oportunidades, con el fin de obtener una estructura que permitiera desarrollar en profundidad la reflexión profesional de los docentes. Esto implicó que el espiral del ciclo de investigación se repitiera cada año, con el fin de otorgarle validez externa e interna al proceso de investigación.

Este proceso consideró las siguientes fuentes de recolección de datos:

1. Observación de campo del desarrollo de los talleres de escritura y evaluación, por parte del equipo, del desempeño alcanzado por cada cohorte en las crónicas.

2. Entrevistas aplicadas a un grupo de estudiantes de cada cohorte, con el fin de obtener sus apreciaciones personales respecto del proceso de escritura de la crónica.

3. Filmación de algunas sesiones de retroalimentación personal y posterior análisis de estas por el equipo del programa.

4. Recogida del producto final (última versión después de dos borradores) que escribían los estudiantes, y conformación de un corpus de 160 textos escritos correspondiente a la crónica docente-directiva.

5. Validación, por juicio de experto, de la organización retórica final de la crónica docente-directiva.
La escritura de las crónicas de cada uno de los estudiantes de programa de posgrado fue una actividad curricular clave del proceso formativo; por ello, se ejecutó una secuencia didáctica que los apoyara durante todo el proceso de producción escrita.

El modelo que subyace a la implementación de esta secuencia didáctica tiene sus fundamentos en la pedagogía del género (Chaisiri, 2010; Hyland, 2007), pues se diseñó desde una concepción constructivista del aprendizaje, que pone énfasis en el andamiaje pedagógico de la escritura de la crónica, siendo fundamental la retroalimentación (feedback) dialógica (Carless, 2015; Jolly y Boud, 2015) y la evaluación de la escritura progresiva (Hawe y Parr, 2014).

La secuencia didáctica que se muestra en la figura 2 enfatiza en la importancia de los borradores, la retroalimentación de ellos y en el rol del tutor de escritura. Al trabajar con estos elementos de forma sistemática, es posible incidir directamente no solo en el andamiaje de la habilidad de la escritura, sino también en la incorporación de nuevos conocimientos lingüísticos, en el desarrollo de una reflexión pedagógica más profunda y, sobre todo, en la autoeficacia del escritor consigo mismo, en tanto se siente más seguro para avanzar en la producción del texto.

Además, este proceso promueve la actividad metacognitiva, el desarrollo de la autonomía y la autorregulación en el aprendizaje, características que ayudan a comprender mejor el objetivo de aprendizaje, el estado de sus logros y los caminos que el profesor debe implementar para acortar sus distancias y obtener logros significativos.

\section{Resultados}

En este apartado presentamos los resultados de la investigación de acuerdo con los objetivos planteados. Así, el objetivo específico 1 permitió consolidar un nuevo género de reflexión pedagógica, denominado "crónica del docente-directivo".

Este género forma parte de la familia de géneros de reflexión profesional docente y tiene como propósito 
comunicativo reflexionar en torno a la experiencia del propio docente o directivo, respecto de aquellos acontecimientos o situaciones que han generado una disonancia cognitiva durante su quehacer o desempeño profesional.

Las principales modalidades discursivas implicadas en este género son la narración y la argumentación. La primera permite identificar y describir la disonancia cognitiva del docente desde una perspectiva subjetiva que moviliza su imperativo moral, activando diversos niveles de reflexión. La segunda le posibilita explicar los nudos críticos de la disonancia cognitiva y justificarlos a la luz de la bibliografía especializada, apoyándose en citas y referencias provenientes de autores e investigaciones destacadas en su ámbito de acción.

Al ser un género que circula en un programa de posgrado, también presenta un carácter académico evaluativo (Jarpa, 2014), pues el contexto de producción donde surge la crónica está asociado a un trabajo final, que es calificado y que certifica un desempeño específico. Esto condiciona el objetivo de aprendizaje de la tarea de escritura, que es analizar, en profundidad, la experiencia profesional del propio docente o directivo, de manera tal que pueda distinguir sus nudos críticos y examinarlos de acuerdo con el nuevo conocimiento adquirido en el programa de posgrado. La delimitación de este objetivo de aprendizaje permite establecer con claridad las estrategias de enseñanza y las técnicas y los criterios de evaluación apropiados.

Para alcanzar el objetivo específico 2, describir la organización retórica del género de reflexión pedagógica, mediante la identificación de los segmentos textuales que lo configuran, se llevaron a cabo las siguientes acciones: en primer lugar, se establecieron determinados segmentos textuales (movidas), con el fin de orientar la escritura de los docentes. En segundo lugar, se analizaron los textos producidos, para ver la pertinencia de las movidas y evaluar el grado de complejidad para alcanzar la organización retórica propuesta y la incorporación de nuevos conocimientos lingüísticos para comunicar de forma más eficiente esta reflexión pedagógica.
Finalmente, se ajustó la nueva propuesta y se evaluó por juicio de expertos. Esta triangulación de los datos permitió consolidar la estructura que se presenta en la tabla 1 .

A continuación se presentan extractos del corpus, ${ }^{1}$ para ejemplificar la forma como los docentes construyen cada movida de la crónica y cómo dan cuenta de esta reflexión pedagógica, mediante la incorporación de ciertos recursos lingüísticos presentados en el módulo de escritura académica.

La movida 1, Cautivar al lector, busca generar una impresión favorable hacia el lector, mediante la incorporación de un breve relato que dé cuenta de una situación particular, que puede tener características emotivas y que, por lo tanto, apele a la complicidad y la empatía del lector.

Santiago, 18 de Junio 2009.

— “Aló, Director, venga pronto. ¡Se están tomando el Colegio!”. Era el nochero que me despertaba con esa alerta a las $6.30 \mathrm{am}$.

Me vestí a toda velocidad y corrí a la portería.

Efectivamente, unos jóvenes de colegios públicos de Puente Alto habían intentado poner cadenas al portón de ingreso y colgar unos lienzos protestando contra la segregación del sistema escolar. El Colegio en cuestión era un colegio particular pagado y de orientación católica marcado por un fuerte compromiso social y promoción de la justicia.

La acción fue impedida por dos inspectores que siempre llegan temprano, quienes obligaron a los intrusos a despejar la entrada y replegarse en la vereda del frente. Ahí se pusieron a mostrar sus pancartas y gritar sus consignas: "Educación de calidad para todos"; "Queremos equidad". "Mi papá gana lo que cuesta tu mensualidad" (CR012016). ${ }^{2}$

1 Los textos se transcriben tal cual los originales. Solo se hacen mínimos cambios editoriales.

2 La referencia CR072013 corresponde a la codificación de los textos del corpus, donde CR es el género, 07 el número del texto, y 2013, el año en que se escribió. Cada una de estas codificaciones cambia según el año de la recolección de los textos. 
Tabla 1 Organización retórica de la crónica docente-directiva

\begin{tabular}{|c|c|c|}
\hline $\begin{array}{l}\text { Organización } \\
\text { estructural }\end{array}$ & Movida retórica & Descripción operacional \\
\hline \multirow[t]{3}{*}{ Introducción } & 1. Cautivar al lector & $\begin{array}{l}\text { Generar una impresión favorable hacia el lector, que implique involucrarlo en el } \\
\text { desarrollo del relato desde una perspectiva cercana. }\end{array}$ \\
\hline & 2. Contextualización del relato & $\begin{array}{l}\text { Presentar y describir el lugar físico, pero también las condiciones ambientales } \\
\text { (subjetivamente) que circundan el relato, el tiempo histórico y la presentación } \\
\text { del autor. Se puede agregar características relevantes de la institución educativa } \\
\text { que aporten a la comprensión global de la situación. }\end{array}$ \\
\hline & 3. Disonancia cognitiva & $\begin{array}{l}\text { Explicitar el quiebre que se provoca como consecuencia de la diferencia que hay } \\
\text { entre el quehacer pasado y el futuro. }\end{array}$ \\
\hline \multirow[t]{3}{*}{ Desarrollo } & 4. Imperativo moral & $\begin{array}{l}\text { Exponer las razones éticas y morales que provocan la disonancia y que } \\
\text { movilizan el actuar y las preocupaciones del narrador. }\end{array}$ \\
\hline & 5. Discusión bibliográfica & $\begin{array}{l}\text { Justificar la disonancia cognitiva desde diversas perspectivas provenientes de } \\
\text { la literatura especializada, con el fin de generar un análisis y comprensión } \\
\text { profunda. }\end{array}$ \\
\hline & $\begin{array}{l}\text { 6. Reflexión sobre las acciones de la } \\
\text { disonancia cognitiva }\end{array}$ & Evaluar el propio actuar del docente en relación con las decisiones tomadas. \\
\hline Conclusión & 7. Desafío transformacional & $\begin{array}{l}\text { Explicitar los cambios realizados, los que se están realizando o los que se } \\
\text { realizarán a partir del imperativo moral puesto en juego en la disonancia } \\
\text { cognitiva. }\end{array}$ \\
\hline
\end{tabular}

En esta movida, los profesores suelen incorporar secuencias dialogales (Adam, 1990), con el fin de hacer del relato algo más cercano a la realidad y así recuperar el tiempo, espacio y las personas involucradas. Es frecuente encontrar diálogos con estilo directo, donde es necesario utilizar marcadores gráficos como los dos puntos, las comillas o el uso de guion largo o raya. De la misma manera, también se emplean diálogos con estilo indirecto, introducidos mediante la conjunción "que". Al cambiar del estilo directo al estilo indirecto, a veces cambian también los tiempos verbales, los pronombres y otras formas lingüísticas, para establecer correctamente la concordancia.

La movida 2, Contextualización del relato, describe la circunstancia donde se enmarca la disonancia cognitiva. Este apartado es de gran importancia, pues permite comprender el alcance e impacto de los conflictos profesionales vividos por los docentes.

Mi escuela municipal, está ubicada en Viña del Mar, es una construcción nueva, diseñada para acoger a la diversidad, con amplios accesos, rampas y ascensor, un lujo que no cualquier escuela tiene. Contamos con un Proyecto de Integración Escolar (PIE), que tiene cuatro profesoras diferenciales de distintas especialidades, una psicopedagoga, dos psicólogos, una asistente social, una fonoaudióloga y una terapeuta ocupacional, todos al servicio de nuestros alumnos.

Cada profesor de asignatura de Lenguaje, Matemática e Inglés cuenta con tres horas a la semana, adicionales para planificar en conjunto con las educadoras diferenciales, lo cual permite coordinar diferentes estrategias y metodologías.

Todo esto con una gran conciencia de las características de vulnerabilidad social de nuestros alumnos y alumnas (CR072013).

Para la construcción de esta movida se adoptan secuencias textuales predominantemente descriptivas, pues el foco está puesto en lograr representar, de la mejor forma posible, las condiciones contextuales en que se desarrolla la disonancia. Por lo mismo, es frecuente que los profesores utilicen formas verbales de aspecto imperfectivo, que indican suspensión temporal. También se emplean marcadores textuales para estructurar u organizar los elementos que configuran la descripción. Desde el 
punto de vista más sintáctico, existe la tendencia al uso de oraciones coordinadas y yuxtapuestas. En estos casos, la ausencia de elementos conectores permite dar mayor viveza a lo que se describe.

La movida 3, Disonancia cognitiva, está vinculada directamente a un incidente crítico que gatilla un cuestionamiento profundo respecto del rol y la responsabilidad profesional del docente en la situación antes descrita, definiendo un antes y un después del ejercicio profesional.

Llegué a la empresa, me presenté con la secretaria, para que me comunicara con el jefe y poder conversar sobre el desempeño del estudiante. Paso a hablar con él, me saluda y lo primero que me dice, “¿ por qué profesora me mandó un alumno tan tonto?” En ese milésimo de segundo, cuando me trasmitió su mensaje, quedé pasmada. Uno nunca espera una respuesta de esa envergadura. Mis primeras palabras fueron, “¿qué pasó don Cristian?. Entonces, percibí que en realidad el estudiante no era capaz de hacer individualmente las labores encomendadas, pero sí hacía sus deberes como correspondía cuando tenía un trabajador apoyándolo. Esto provocó que en forma permanente estuviera una persona con él, por lo cual la empresa tuvo que disponer de un tutor a tiempo completo, implicando un gasto extra que no lo tenían contemplado.

$\mathrm{Al}$ regresar al establecimiento, entré a mi oficina, me senté e hice una autoevaluación con el objetivo de solucionar esos nudos críticos, y con la finalidad de mejorar la inserción laboral de cada estudiante. Tuve temor de contar inmediatamente lo acontecido porque de alguna manera me iban a juzgar, pero pasaron algunos minutos y me acerqué al inspector, porque sabía que me iba a entregar un consejo constructivo. Lo primero que me preguntó: “'ipreparaste al empresario sobre este tema? ¿Analizaste en la reunión técnica cual era el mejor Centro de Práctica para él, considerando sus competencias y capacidades? ¿Se preparó al alumno sobre las funciones en que se iba a desempeñar en la empresa?", todas las interrogantes las fui contestando a medida que íbamos profundizando sobre el tema. Fue difícil reconocer que hubo vacíos, ya que si todo hubiese estado bien, nada de esto hubiese ocurrido (CR052014).

Esta movida se construye fundamentalmente con secuencias narrativas, ya que un aspecto relevante es la configuración del punto de vista que el autor decide utilizar para exponer su disonancia cognitiva.
De la misma manera, hay una organización temporal, donde el narrador se hace parte del relato para dar cuenta de sus pensamientos o de las acciones de los participantes implicados. También se utilizan formas verbales de presente y del pretérito perfecto simple e imperfecto. Por otra parte, se emplean marcadores u organizadores textuales de tipo temporal o que expresen algún tipo de secuencialidad: "lo primero", "en ese momento", “entonces”, “después”, etc.

La movida 4, Imperativo moral, se desprende directamente de la identificación de la disonancia, pues surgen interrogantes y cuestionamientos éticos y morales que movilizan el actuar de los docentes y provocan un cambio en la proyección profesional de ellos.

En mi cabeza fluyen pensamientos como: muchos padres y madres no podrán organizarse con tan poco tiempo de aviso; un día sin asistencia nos liquida (por la subvención) para poder cancelar sueldos a fin de mes; tengo horas para organizar el colegio, y mientras pensaba los invitaba a negociar alguna forma de no detener la escuela y poder ser parte del proceso de movilización (el cual desde mi propia mirada merecía un espacio serio de atención, había ya leído varios análisis de la ley y entendía que estábamos inmersos en un proceso social que nos tocaba con fuerza, por tanto era importante estar) mis sugerencias terminaban en silencio. Decididos y decididas argumentaban que estaban dispuestos y dispuestas a recibir el descuento correspondiente, que era un derecho y que la escuela no era una guardería, por último señalaron que la prontitud del aviso se debía a la contingencia misma (CR082015).

De igual manera, en esta movida predominan las secuencias dialogales, como monólogos interiores, soliloquios e incluso corriente de conciencia, la que se caracteriza por ser una variedad monológica de reproducción del pensamiento en forma indirecta, en la que, desde una focalización omnisciente, el narrador relata con su propia voz una perspectiva psíquica de su vida o la de otros que participan de la situación. Con estos recursos, el profesor busca reproducir sus mecanismos de pensamiento en el texto y así poder explicitar percepciones internas asociadas a lo ético y lo moral de la disonancia. La estructura del monólogo interior 
depende del grado de conciencia del protagonista; por lo mismo, requiere de un lenguaje especial, muchas veces, sin puntuación, con juegos verbales y sintácticos.

La Discusión bibliográfica corresponde a la movida 5 y es el segmento textual donde se debe poner en evidencia el dominio disciplinar adquirido durante el programa de posgrado. Para ello resultan relevantes los recursos de atribución de conocimiento que se utilizan y la forma como se tensionan y contrastan la experiencia profesional y las propuestas teóricas de los diversos ámbitos disciplinares de la especialidad adquirida en el posgrado.

Aún hoy en día existe resistencia por parte de algunos profesores al cambio. Muchos de ellos se sienten inseguros en cuanto a sus capacidades para enfrentarse a niños con ciertas dificultades de aprendizaje y emocionales, sienten que no han sido suficientemente preparados o "no estudiaron para eso". Sobre esto hablan Tournaki y Podell (2005), cuando se refieren a las "creencias positivas hacia la inclusividad que deben tener los profesores, ya que muchos de ellos creen que tienen insuficientes recursos para implementar la inclusividad exitosamente".

Si consideramos lo anterior, y logramos entender que la educación no sólo debe respetar el derecho a ser diferentes, sino que debe valorar en forma explícita lo enriquecedor que resulta para la comunidad educativa la diversidad en las aulas, podremos vivir lo que es una escuela inclusiva, donde los profesores, los alumnos y los padres convivan entre ellos, tengan o no capacidades diversas o sean parte de una raza, cultura o religión diferente (CR022018).

En esta movida predomina el uso de la secuencia expositivo-explicativa, pues se presenta la exposición de un saber construido por otros y que está legitimado socialmente, o también la exposición de un saber teórico referido al ámbito de los hechos que asume la forma de juicio constatativo. Por lo mismo, esta movida incorpora recursos discursivos de heteroglosia, para otorgarle sustento teórico especializado al análisis de la disonancia. Así, en este segmento, la atribución de conocimiento se realiza mediante dos mecanismos: por una parte, la citación, donde el escritor recupera información proveniente de otras fuentes especializadas, y, por otra, las marcas de inscripción de la primera persona, que sirven para analizar cómo se incluye la voz propia del escritor respecto de las otras voces que admite.

No obstante lo anterior, algunos textos tienden a borrar las huellas del sujeto enunciador (marcas valorativas, afectivas o apreciativas) e instaurar distancia respecto de lo citado, para generar el efecto de objetividad.

En cuanto a la movida 6, Reflexión sobre las acciones de la disonancia cognitiva, esta permite desarrollar un análisis respecto del propio actuar y las implicancias a nivel institucional, grupal e individual.

En el caso de la institución a la cual dirijo, la posibilidad de contar con una relación directa y cercana con la sostenedora del establecimiento, ha permitido que como organización hayamos comenzado a delinear prácticas institucionales orientadas a generar condiciones y posibilidades de desarrollo personal y profesional de los funcionarios, tarea que si bien está recién en proceso de iniciación, da cuenta de una intención por dar vida a un concepto integral de comunidad educativa (CR092014).

Desde el punto de vista de la secuencia textual predomina la argumentación, pues el foco está en situar una perspectiva de análisis para la disonancia y el impacto de esta en la experiencia profesional del docente. También se utilizan recursos semánticodiscursivos para valorar o evaluar la experiencia, expresando apreciaciones y sentimientos desde una perspectiva inter e intrapersonal.

Finalmente, el Desafio transformacional, correspondiente a la movida 7 de la organización retórica de la crónica, proyecta acciones de cambio que permitan cualificar el ejercicio profesional y avanzar hacia nuevas oportunidades de mejora, tanto a nivel institucional como individual.

Hoy, estoy utilizando herramientas de gestión y liderazgo que me han servido para mejorar mis competencias directivas. Tengo clara mi meta a lograr haciendo operativo el contenido del PEI [Proyecto Educativo Institucional] con la misión y visión, enfatizar el sello que nos distingue como Escuela, en este caso es el sello de formación artístico cultural. Este desafío no es fácil, 
pues implica una transformación en toda la cultura escolar, me motiva la inquietud y las ganas que visualizo en los docentes y toda la comunidad de esta escuela, hay que dar el paso, de una escuela que enseña a una escuela que aprende (CR082016).

Esta movida recurre a segmentos textuales narrativos y descriptivos para mostrar acciones presentes o futuras que dan cuenta del cambio en los modos de pensar y actuar de los docentes. Para ello se emplean formas verbales del presente, pues indican que las acciones no han terminado o se están realizando. Asimismo, se puede utilizar el condicional simple, que permite enunciar gestiones venideras, cercanas al tiempo actual, o bien el futuro imperfecto, para exponer las acciones como obligaciones o propósitos, como convicciones de certezas o con valor de duda (Grijelmo, 2017).

Cada una de las movidas retóricas antes descritas organizan los contenidos de la crónica, permitiendo una articulación lógica entre la experiencia del profesor y los conocimientos especializados que este debe tener para alcanzar un nivel de reflexión profundo que le permita construir y consolidar su saber pedagógico.

Como se mencionó anteriormente, la aplicación de la secuencia didáctica instala un acompañamiento sistemático durante todo el proceso de escritura, incluyendo uno, dos o tres borradores, según sea el caso de cada profesor, es decir, según presente mayores dificultades en los procesos de retroalimentación.

Al finalizar el proceso formativo, se aplica una rúbrica de evaluación sumativa, que consta de cuatro niveles de desempeño (destacado, competente, básico e insuficiente) y de siete criterios de evaluación (construcción de la disonancia; contextualización de la disonancia; modelo de reflexión; atribución de conocimiento; coherencia y cohesión textual; nivel de reflexión y aspectos formales).

Los resultados generales revelan que el $70 \%$ de los textos del corpus alcanzan la categoría de "destacado", lo que significa que el desarrollo de los contenidos a lo largo del texto se presenta de forma coherente, sin contradicciones en la redacción de sus enunciados, y además utilizan diversos recursos de cohesión, lo que posibilita una comprensión acabada del fenómeno planteado. Así mismo, la crónica alcanza un nivel de reflexión crítico y comparativo, logrando integrar a lo largo del texto un análisis profundo de la disonancia cognitiva, con la predominancia de secuencias textuales argumentativas y explicativas. La incorporación de las citas permite identificar una relación dialógica entre la propia reflexión y el conocimiento referido desde diversas fuentes.

Por su parte, solo un $20 \%$ de los textos son clasificados en el nivel de desempeño "competente", es decir, textos que si bien presentan un buen nivel comunicativo, las dificultades radican en que la naturaleza de la reflexión es predominantemente descriptiva y el uso de fuentes solo alcanza un nivel relacional. En cuanto al $10 \%$ restante de los textos del corpus, un $6 \%$ es categorizado como "básico" y solo un $4 \%$ como "insuficiente".

Sin duda, el al to porcentaje de logro obtenido es posible gracias al modelo de intervención didáctica, que tiene como centro el modelamiento de la escritura basado en la pedagogía del género, lo que implica, a su vez, el uso de borradores y retroalimentación permanente, tanto del tutor experto como de los pares.

\section{Conclusiones}

A lo largo de este artículo se analizó la importancia de la escritura en la formación de profesores y directivos docentes, se describió la organización retórica de un nuevo género al que denominamos "crónica docente-directiva" y sus recursos lingüísticos, y se describió la secuencia didáctica desarrollada con los docentes en los talleres de escritura.

Dados estos antecedentes, una primera conclusión es que la crónica del docente-directivo es una herramienta formativa, con madurez didáctica, que permite unir teoría y práctica, y potencia el saber pedagógico y la trayectoria profesional de los profesores.

La interrelación entre dos perspectivas teóricas y metodológicas, como lo son la teoría del género y la 
pedagogía del género, permitieron a los participantes del programa desarrollar un proceso reflexivo profundo, basado en su experiencia profesional y en el conocimiento especializado, pues los incitó a poner en el centro de la discusión sus propias prácticas.

De la misma manera, la organización retórica de la crónica tiene una progresión en la complejidad de las secuencias discursivas utilizadas en las diversas movidas. Así, las primeras (movida 1 a la 3 ) se construyen con base en secuencias dialogales, descriptivas y narrativas, mientras que las finales (movida 4a la 7) dan cuenta del análisis y la reflexión de ejercicio profesional, y requieren secuencias argumentativas y algunos recursos lingüísticos de modalización, modulación, de monoglosia y heteroglosia para alcanzar una reflexión profunda.

Facilitar y estimular la reflexión sobre las prácticas directivas y docentes dependen de dos aspectos fundamentales: por una parte, que los docentes vean, en este ejercicio de escritura, una oportunidad de análisis individual y colectivo de la disonancia cognitiva; $y$, por otra, que cuenten no solo con una variedad de aproximaciones bibliográficas que les permitan juzgar la diversidad de aristas del fenómeno crítico, sino también con recursos semánticos discursivos y lingüísticos, para poder mirar y analizar el fenómeno pedagógico en su más amplio espectro.

Desde una perspectiva del discurso especializado, la crónica docente-directiva entreteje una sutil y consistente malla conceptual, nutrida por diversas disciplinas (pedagogía, psicología, ingeniería, derecho), que permiten reinterpretar la disonancia cognitiva en búsqueda de nuevos caminos de acción que vinculen fundamentos teóricos con acciones prácticas. Esto demanda una aproximación multidisciplinaria, en donde el escritor de la crónica acude a diferentes teorías, explicaciones y autores para desarrollar su reflexión. Cada participante deliberadamente selecciona un tema específico, la literatura que utilizará y los puntos explicativos de conexión. En este proceso es acompañado por el tutor de escritura, quien retroalimenta los borradores y realiza entrevistas personales con cada uno, para discutir el avance del texto y cautelar la comprensión del proceso que se lleva a cabo.

De la misma manera, las interrelaciones conceptuales creadas por los escritores de las crónicas directivas demandan, del equipo de profesores del programa de Magíster, un diálogo permanente para comprender nuevos puntos de encuentro, y estar abiertos al trabajo colegiado auténtico y a cuestionar y repensar el propio proceso didáctico. Esta perspectiva abre otra dimensión, que guarda relación con los procesos de cualificación continua que desarrolla el programa de posgrado, pues la metodología de investigación-acción y la escritura académica han significado una oportunidad para identificar dificultades y desarrollar acciones que mejoren la capacidad del equipo y los resultados formativos de los participantes.

\section{Referencias}

Adam, J. M. (1990). Éléments de linguistique textuelle. Théorie et pratique de l'analyse textuelle. Lieja: Mardaga.

Anderson, L., y Krathwohl, D. (Eds.) (2001). A Taxonomy for Learning, Teaching, and Assessing: A Revision of Bloom's Taxonomy of Educational Objectives. New York: Addison Wesley Longman.

Bazerman, Ch. (2004). Speech acts, genres, and activity systems: How texts organize activity and people. En Ch. Bazerman y P. Prior (Eds.), What Writing does and how it does it. An Introduction to Analyzing Texts and Textual Practice (pp. 309-340). London: LEA.

Bereiter, C., y Scardamalia, M. (1987), The Psychology of Written Composition. Hillsdale, Erlbaum.

Bermúdez, N. (2009). Escritura y producción de conocimiento en las carreras de postgrado. Santiago de Chile: Arcos.

Bhatia, V. (1993). Analysing Genre-Language Use in Professional Settings. London: Longman.

Carless, D. (2015). La confianza: facilitar el feedback dialógico. En D. Boud y E. Molloy (Coords.), El feedback en educación superior y profesional. Comprenderlo y hacerlo bien (pp. 115-129). Madrid: Narcea.

Carlino, P. (2006). Escribir, leer y aprender en la universidad. Buenos Aires: Fondo de Cultura Económica.

Cassany, D. (2006). Tras las líneas. Sobre la escritura contemporánea. Barcelona: Anagrama, S. A. 
Chaisiri, T. (2010). Implementing a genre pedagogy to the teaching of writing in a university context in Thailand. Language Education in Asia, 1(1), 181-199. https://doi.org/10.5746/leia/10/v1/a16/chaisiri

Cotos, E., Huffman, S., y Link, S. (2017). A move/step model for methods sections: Demonstrating rigor and credibility. English for Specific Purposes, 46, 90-106. https://doi.org/10.1016/j.esp.2017.01.001

Darling-Hammond, L. (2006). Power Teacher Education. Lessons from Exemplary Programs. San Francisco: Jossley Bass.

Darling-Hammond, L. (2012). Educar con calidad y equidad. Los dilemas del siglo XX. Santiago: Centro de Innovación en Educación de Fundación Chile.

Elliott, J. (1993). El cambio educativo desde la investigaciónacción. Madrid: Morata.

Festinger, E. (1957). A theory of Cognitive Disonance. Stanford: Stanford University.

Grijelmo, A. (2017). La gramática descomplicada. Barcelona: Taurus.

Hatton, N., y Smith, D. (1995). Reflection in teacher education: Towards definition and implementation. Teaching \& Teacher Education, 11(1), 33-49. https://doi.org/10.1016/0742-051x(94)00012-u

Hyland, K. (2003), Genre-based pedagogies: A social response to process. Journal of Second Language Writing, 12(1), 17-29. https://doi.org/10.1016/ s1060-3743(02)00124-8

Hyland, K. (2007). Genre pedagogy: Language, literacy and L2 writing instruction. Journal of Second Language Writing, 16, 148-164. https://doi.org/10.1016/j. jslw.2007.07.005

Jarpa, M. (2012). Macrogénero académico evaluativo: descripción retórica en la comunidad de aprendizaje de postgrado en biotecnología (tesis de doctorado). Pontificia Universidad Católica de Valparaíso, Chile.

Jarpa, M. (2014). Macrogénero académico evaluativo. Descripción retórica de los géneros académicos evaluativos en una comunidad de aprendizaje de postgrado. Sarrebruck: Publicia.

Jay, J. K., y Johnson, K. L. (2002). Capturing complexity: A typology of reflective practice for teacher education. Teaching and Teacher Education, 18(1), 73-85. https://doi.org/10.1016/s0742-051x(01)00051-8

Jolly, B., y Boud, D. (2015). El feedback por escrito. Para qué sirve y cómo podemos hacerlo bien. En D. Boud y E. Molloy (Coords.), El feedback en educación superior y profesional. Comprenderlo y hacerlo bien (pp. 131154). Madrid: Narcea.
Kanoksilapatham, B. (2007). Introduction to move analysis. En D. Biber, U. Connor y T. Upton (Eds.), Discourse on the Move (pp. 23-41). Amsterdam: Benjamins.

Kemmis, S., y McTaggart, R. (1988). ¿Cómo planificar la investigación-acción? Barcelona: Laertes.

Korthagen, F. A. J. (2004). In search of the essence of a good teacher: Towards a more holistic approach in teacher education. Teaching and Teacher Education, 20(1), 77-97. https://doi.org/10.1016/j. tate.2003.10.002

Kress, G., y Van Leeuwen, T. (2001). Multimodal Discourse. The Modes and Media of Contemporary Communication. Londres: Arnold.

Lewin, K. (1973). Action research and minority problems. En K. Lewin (Ed.), Resolving Social Conflicts: Selected Papers on Group Dynamics (pp. 201-216). Londres: Souvenir Press.

Parodi, G., Ibáñez, R., Venegas, R., y González, C. (2010). Identificación de géneros académicos y géneros profesionales: principios teóricos y propuesta metodológica. En G. Parodi (Ed.), Alfabetización académica y profesional en el siglo XXI: leer y escribir desde las disciplinas (pp. 249-289). Santiago: Ariel.

Parr, J., y Hawe, E. (2014). Assessment for learning in the writing classroom: An incomplete realisation. The $\mathrm{Cu}$ rriculum Journal, 25(2), 210-237. http://dx.doi.org $/ 10.1080 / 09585176.2013 .862172$

Quintero, J., Torres, F., y Cardona, M. (2007). El maestro escribe su saber y su hacer. Lenguaje, 35(1), 279-300.

Schön, D. (1998). El profesional reflexivo: cómo piensan los profesionales cuando actúan. Barcelona: Paidós Ibérica.

Starks, D., Nicholas, H., y Macdonald, S. (2012). Structured reflective communication as a meta-genre in teacher education: Creative uses of critique in a teacher education program. Australian Journal of Teacher Education, 37(3), 90-111. http://dx.doi. org/10.14221/ajte.2012v37n3.8

Swales, J. (2000). Genre Analysis. English in Academic and Research Settings. Cambridge: Cambridge University Press.

Thaiss, C., y Myers, T. (2006). Engaged Writers and Dynamic Disciplines: Research on the Academic Writing Life. Boynton/Cook: Heinemann Press.

Venegas, R., Núñez, M., Zamora, S., y Santana, A. (2015). Escribir desde la pedagogía del género. Valparaíso: Ediciones Universitarias de Valparaíso.

Villalobos, J., y De Cabrera, C. (2009). Los docentes y su necesidad de ejercer una práctica reflexiva. Revista de Teoria y Didáctica de las Ciencias Sociales, (14), 139-166. 
Williamson, G. (2002). Investigación acción participativa intercultural en comunidades educacionales y locales. Temuco: Universidad de La Frontera/Proyecto Kelluwün.

Yinger, R. J., y Clark, C. M. (1981). Reflective Journal Writing: Theory and Practice. (Occasional Paper series,
N. ${ }^{\circ}$ 50). East Lansing, MI: Michigan State University, Institute for Research on Teaching.

Zuluaga, O., y Echeverri, A. (2003). El florecimiento de las investigaciones pedagógicas. En: Pedagogía y epistemología (pp. 57-80). Bogotá: Grupo Historia de Práctica Pedagógica-Cooperativa Editorial Magisterio.

How to reference this article: Jarpa-Azagra, M. (2019). Escritura académica para el desarrollo de la reflexión pedagógica en la formación docente: la crónica del docente-directivo. Íkala, Revista de Lenguaje y Cultura, 24(1), 85-101. Dor: 10.17533/udea.ikala.v24n01a04 